\title{
Erratum: Spatiotemporal bounded noises and transitions induced by them in solutions of the real Ginzburg-Landau model [Phys. Rev. E 86, 021118 (2012)]
}

Sebastiano de Franciscis and Alberto d'Onofrio

(Received 28 October 2016; published 10 November 2016)

DOI: 10.1103/PhysRevE.94.059905

In the original paper, we introduced two spatiotemporal colored bounded noises, one of which was based on the zerodimensional Cai-Lin noise [1] described by the following stochastic differential equation (SDE):

$$
\xi^{\prime}(t)=-\frac{1}{\tau_{c}} \xi(t)+\sqrt{\frac{1}{\tau_{c}(1+\delta)}} \sqrt{B^{2}-\xi^{2}} \eta(t),
$$

$\eta(t)$ is a white noise. Namely, the spatiotemporal extension reads

$$
\partial_{t} \xi(x, t)=\frac{2 \lambda^{2}}{\tau_{c}} \nabla^{2} \xi(x, t)-\frac{1}{\tau_{c}} \xi(x, t)+\sqrt{\frac{2 D}{\tau_{c}(1+\delta)}} \sqrt{B^{2}-\xi^{2}} \eta(x, t) .
$$

Recently, we discovered that Doering had previously defined, in a 1987 paper [2] (see also Ref. [3]), both Cai-Lin equation Eq. (1) and our Eq. (2). Luckily, no other overlap exists between our paper and the paper [2] which investigated very interesting analytical properties of the model (2) in the one-dimensional case. On the contrary, in the original paper we numerically studied the properties of the two spatiotemporal noises in a two-dimensional (2D) lattice approximation as well as their impact on the solutions of the Ginzburg-Landau model, again in 2D. It is a pleasure to recognize the priority of Doering in proposing model 2. Finally, in the Appendix of the original paper, in a section on a method to generate bounded noises with a preassigned stationary density from a scalar stochastic differential equation with additive noise, we mentioned en passant that, if the preassigned stationary density is $P(x)=(1 / 2) \cos (x)_{+}$, then the drift of the generating SDE is proportional to $-\tan (x)$. This process (which had no role in our paper, apart from being an example of a general method we proposed) has been defined and investigated in Ref. [4], which we did not know when we wrote our paper.

[1] G. Q. Cai and Y. K. Lin, Phys. Rev. E 54, 299 (1996).

[2] C. R. Doering, Phys. Lett. A 122, 133 (1987).
[3] C. R. Doering, Phys. Lett. A 344, 149 (2005).

[4] M. Kessler and M. Sørensen, Bernoulli 5, 299 (1999). 